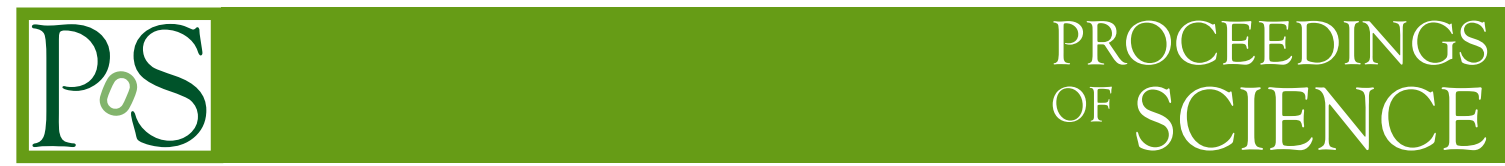

\title{
CEvNS nuclear physics aspects
}

\section{Dimitrios K. Papoulias ${ }^{a, *}$}

${ }^{a}$ Division of Theoretical Physics, University of Ioannina, GR 45110 Ioannina, Greece

E-mail: d.papoulias@uoi.gr

The recent observation of coherent elastic neutrino-nucleus scattering (CEvNS) has opened new opportunities for investigating nuclear structure parameters and other electroweak probes. Here, we review the implications of advanced nuclear structure models, such as the Deformed Shell Model on the interpretations of COHERENT data.

*** Particles and Nuclei International Conference - PANIC2021 ***

*** 5 - 10 September, $2021 * * *$

*** Online ***

${ }^{*}$ Speaker 


\section{Introduction}

Existing uncertainties on the finite nuclear size are limiting the constraining power of experimental searches based on nuclear recoil measurements. Characteristic such examples include several experimental probes looking for coherent elastic neutrino-nucleus scattering (CEvNS) and direct detection of dark matter. In theoretical simulations of the expected event rates, the nuclear physics dependence is typically incorporated through the nuclear form factors defined as the Fourier transform of the nuclear charge density distribution [1]

$$
F_{n, p}\left(Q^{2}\right)=\frac{1}{N_{a}} \int \rho_{p, n}(\vec{r}) e^{i \vec{Q} \cdot \vec{r}} d^{3} \vec{r}, \quad N_{a}=Z, N,
$$

with $F_{p} \neq F_{n}$ and $Q$ being the magnitude of the 3-momentum transfer. The $k$-th radial moment of the charge density distribution can be cast in the form

$$
\left\langle R_{p, n}^{k}\right\rangle=\frac{\int \rho_{p, n}(\vec{r}) r^{k} d^{3} \vec{r}}{\int \rho_{p, n}(\vec{r}) d^{3} \vec{r}},
$$

with the most relevant phenomenological parameter in the latter experiments being the so-called nuclear root mean square (rms) radius which corresponds to $k=2$.

From the perspective of experimental physics, the proton charge density distribution has been measured with high precision from electron scattering data [2], while the neutron distribution remains still unconstrained. On the other hand, in the framework of advanced nuclear physics methods such as the large-scale Shell-Model [3, 4], the Quasiparticle Random Phase Approximation (QRPA) [5], Microscopic Quasiparticle Phonon Model (MQPM) [6] and the method of Deformed Shell Model (DSM) calculations [7], the proton and neutron nuclear form factors can be treated separately. By relying on the DSM, in this work our main goal is to offer reliable simulations of the expected signal at the COHERENT experiment [8].

\section{DSM and phenomenological nuclear form factors}

In the context of the DSM, the calculation of the nuclear wave functions is implemented in various steps [9]. First, by assuming axial symmetry a Hartree-Fock (HF) calculation is performed for obtaining the lowest intrinsic solution, while the effective interaction is obtained by renormalizing the CD-Bonn potential. The next step involves calculations of the excited intrinsic states based on particle-hole excitations over the lowest intrinsic states. In the final step, angular momentum projection and band mixing calculations are performed for obtaining the final nuclear wave functions. To validate the reliability of the nuclear wave functions, the number of intrinsic states is selected such that important isotopic properties are accurately reproduced, e.g. magnetic moments and other spectroscopic properties. For the detector materials of COHERENT i.e. ${ }^{127}$ I and ${ }^{133} \mathrm{Cs}$ considered in the present study, important observables and outcomes of the nuclear structure calculations from DSM are given in Ref. [10].

Apart from realistic nuclear structure calculations such as those described in Refs. [3-7], various phenomenological form factor approximations also exist in the literature which can describe the nuclear physics corrections with high reliability. Here, we will focus on three typical examples 
which are expressed in analytical form and depend mainly on the nuclear rms radius. First, the Helm form factor [11]

$$
F_{\text {Helm }}\left(Q^{2}\right)=3 \frac{j_{1}\left(Q R_{0}\right)}{q R_{0}} e^{-(Q s)^{2} / 2},
$$

is a widely used approximation which relies on the convolution of two nucleonic densities, one being a uniform density with cut-off radius, $R_{0}$, (namely box or diffraction radius) characterizing the interior density and a second one that is associated with a Gaussian falloff in terms of the surface thickness, $s$. In Eq.(3), $j_{1}(x)$ represents the 1st-order spherical Bessel function and $s=0.9^{1}$. By adopting a conventional Fermi (Woods-Saxon) charge density distribution, another useful form factor description is given by the symmetrized Fermi (SF) approximation [14]

$$
F_{\mathrm{SF}}\left(Q^{2}\right)=\frac{3}{Q c\left[(Q c)^{2}+(\pi Q a)^{2}\right]}\left[\frac{\pi Q a}{\sinh (\pi Q a)}\right] \times\left[\frac{\pi Q a \sin (Q c)}{\tanh (\pi Q a)}-Q c \cos (Q c)\right],
$$

with $c=1.23 A^{1 / 3}-0.60(\mathrm{fm})$ denoting the half density radius and $a=0.52(\mathrm{fm})$ being the diffuseness parameter. It is worth mentioning that the surface thickness in this case is quantified through the relation $t=4 a \ln 3$. Finally, in the Klein-Nystrand $(\mathrm{KN})$ approximation, the form factor follows from the convolution of a Yukawa potential with range $a_{k}=0.7 \mathrm{fm}$ over a Woods-Saxon distribution, approximated as a hard sphere with radius $R_{A}$ [15]

$$
F_{\mathrm{KN}}=3 \frac{j_{1}\left(Q R_{A}\right)}{Q R_{A}}\left[1+\left(Q a_{k}\right)^{2}\right]^{-1} .
$$

Finally, the rms radius corresponding to the three approximations is given below

$$
\left\langle R^{2}\right\rangle_{\mathrm{Helm}}=\frac{3}{5} R_{0}^{2}+3 s^{2}, \quad\left\langle R^{2}\right\rangle_{\mathrm{SF}}=\frac{3}{5} c^{2}+\frac{7}{5}(\pi a)^{2}, \quad\left\langle R^{2}\right\rangle_{\mathrm{KN}}=\frac{3}{5} R_{A}^{2}+6 a_{k}^{2} .
$$

\section{Results and discussion}

Figure 1 illustrates the proton and neutron nuclear form factors, evaluated in the context of DSM calculations. For the sake of comparison, the form factors obtained using the phenomenological Helm, SF and KN parametrization are also shown. It becomes evident that, in general, $F_{p}=F_{n}$ is not always a valid approximation since minima and maxima of $F_{p}$ and $F_{n}$ occur at different values of the momentum transfer. At this point we are interested to explore the implications of the different form factors to theoretical simulations of the expected CEvNS rates at the COHERENTCsI detector. In Fig. 2 we present the estimated number of events calculated in the context of the DSM as well as by using the Helm, SF and KN form factors. In the left panel, our results are compared with the COHERENT measurement [8]. We conclude that the employed DSM nuclear structure calculations lead to an improved agreement with the COHERENT data in comparison to the adoption of the phenomenological form factors. To quantify the latter, we perform a spectral fit of the COHERENT experimental data based on the $\chi^{2}$ function [8]

$$
\chi^{2}=\min _{\xi, \zeta}\left[\sum_{i=4}^{15} \frac{\left(N_{\text {meas }}^{i}-N_{\text {theor }}^{i}[1+\xi]-B_{0 n}^{i}[1+\zeta]\right)^{2}}{\left(\sigma_{\text {stat }}^{i}\right)^{2}}+\left(\frac{\xi}{\sigma_{\xi}}\right)^{2}+\left(\frac{\zeta}{\sigma_{\zeta}}\right)^{2}\right],
$$

${ }^{1}$ This value follows muon spectroscopy data fitting [13] and improves the matching between the Helm and the symmetrized Fermi form factors [12]. 

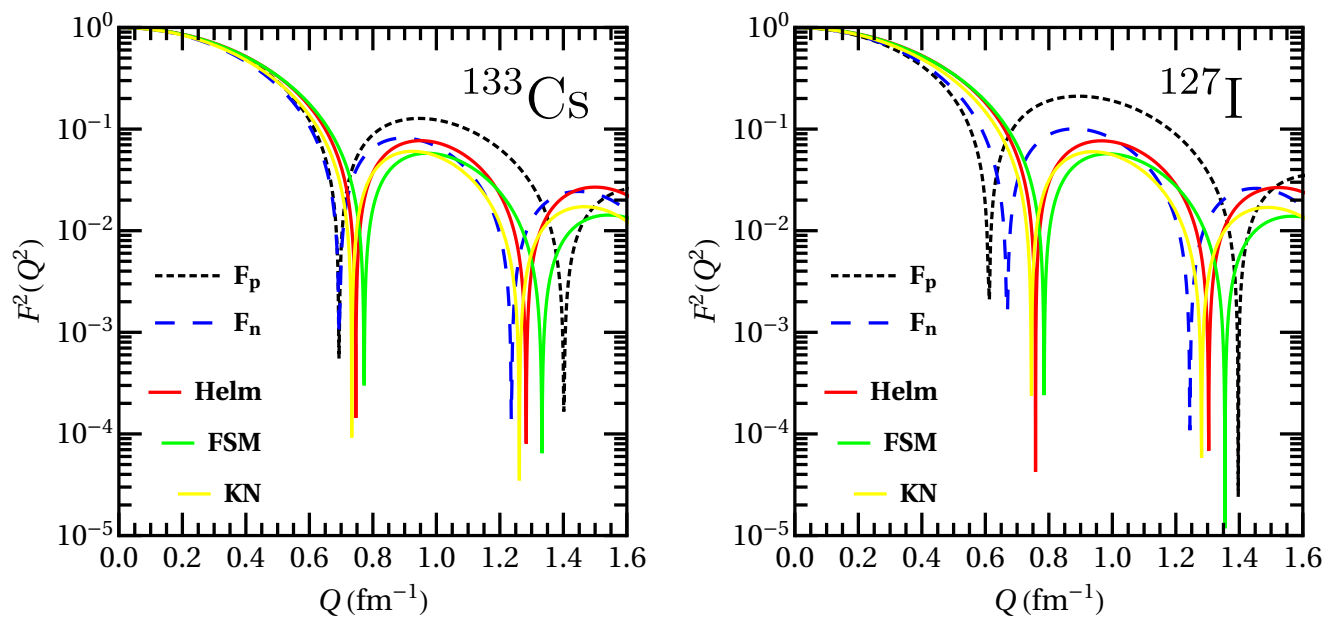

Figure 1: Proton and neutron weak nuclear form factors of ${ }^{133} \mathrm{Cs}$ (left) and ${ }^{127} \mathrm{I}$ (right) nuclei as a function of the momentum transfer $Q\left(\mathrm{fm}^{-1}\right)$, calculated with DSM and compared with Helm, SF and KN form factors, Figure adapted from [10].
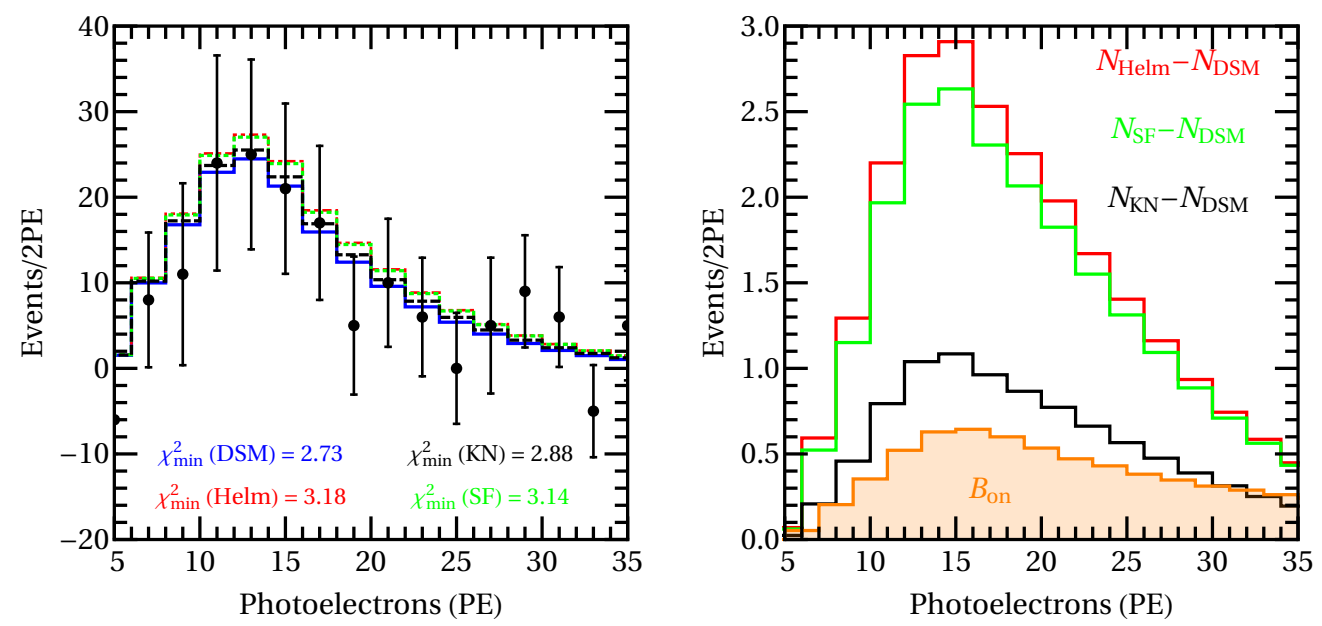

Figure 2: Number of events per 2 photoelectrons at the COHERENT experiment. Left: comparison of the corresponding results calculated with DSM and conventional Helm, SF, KN form factors and the experimental data. Right: difference in events between DSM and phenomenological form factor calculations and Beam-on prompt neutron background events as a function of observed photoelectrons. Figure adapted from Ref. [10].

where $N_{\text {theor }}$ stand for the theoretical number of events (for details see Ref. [10]). Here, $\sigma_{\text {stat }}$ represents the statistical uncertainty, while the nuisance parameters $\xi$ and $\zeta$ account for the signal and background uncertainties $\sigma_{\xi}=28 \%$ and $\sigma_{\zeta}=25 \%$, respectively. From our analysis we find the best fit values $\chi_{\text {min }}^{2}(\mathrm{DSM})=2.73$ compared to $\chi_{\min }^{2}(\mathrm{Helm})=3.18, \chi_{\min }^{2}(\mathrm{SF})=3.14$ and $\chi_{\text {min }}^{2}(\mathrm{KN})=2.88$. Finally, the right panel of Fig. 2 illustrates event differences between calculations based on the DSM and either of the phenomenological form factors, that is $N_{\mathrm{Helm}}-N_{\mathrm{DSM}}, N_{\mathrm{SF}}-N_{\mathrm{DSM}}$ 
and $N_{\mathrm{KN}}-N_{\mathrm{DSM}}$. For comparison, also shown is the distribution of beam-on prompt neutron background events $B_{0 n}$ as a function of the detected photoelectrons. Before closing let us note that the differences in events $N_{\mathrm{Helm}}-N_{\mathrm{SF}}$, although not shown here, are lower than the $B_{0 n}$ level.

\section{Acknowledgments}

The author is thankful to T.S. Kosmas, R. Sahu and V.K.B. Kota for their contributions to the work here presented. This research is co-financed by Greece and the European Union (European Social Fund- ESF) through the Operational Programme «Human Resources Development, Education and Lifelong Learning» in the context of the project "Reinforcement of Postdoctoral Researchers 2nd Cycle" (MIS-5033021), implemented by the State Scholarships Foundation (IKY).

\section{References}

[1] D. K. Papoulias and T. S. Kosmas, Adv. High Energy Phys. 2015 (2015) 763648.

[2] I. Angeli and K. Marinova, Atom.Data Nucl.Data Tabl. 99 (2013) 69-95.

[3] M. Kortelainen, J. Suhonen, J. Toivanen, and T. Kosmas, Phys.Lett.B 632 (2006) 226-232.

[4] P. Toivanen, M. Kortelainen, J. Suhonen, and J. Toivanen, Phys.Rev.C 79 (2009) 044302.

[5] D. Papoulias and T. Kosmas, Phys.Lett. B728 (2014) 482-488.

[6] P. Pirinen, J. Suhonen, and E. Ydrefors, Adv.High Energy Phys. 2018 (2018) 9163586.

[7] D. K. Papoulias, R. Sahu, T. S. Kosmas, V. K. B. Kota, and B. Nayak, ” Adv. High Energy Phys. 2018 (2018) 6031362.

[8] D. Akimov et al. [COHERENT], Science 357 (2017) 1123-1126.

[9] V. K. B. Kota and R. Sahu, Structure of Medium Mass Nuclei: Deformed Shell Model and Spin-Isospin Interacting Boson Model. CRC Press, 2016.

[10] D. Papoulias, T. Kosmas, R. Sahu, V. Kota, and M. Hota, Phys.Lett. B800 (2020) 135133.

[11] R. H. Helm, Phys.Rev. 104 (1956) 1466-1475.

[12] J. Lewin and P. Smith, Astropart.Phys. 6 (1996) 87-112.

[13] G. Fricke et al., Atom.Data Nucl.Data Tabl. 60 (1995) 177-285.

[14] D. W. L. Sprung and J. Martorell, Journal of Physics A: Mathematical and General 30 (1997) 6525-6534.

[15] S. Klein and J. Nystrand, Phys.Rev. C60 (1999) 014903. 